\title{
Effect of ball milling on the local magnetic flux distribution and microstructure of in situ $\mathrm{Fe} / \mathrm{MgB}_{2}$ conductors
}

C. Laliena ${ }^{1}$, T. Qureishy ${ }^{2}$, E. Martínez ${ }^{1, *}$, R. Navarro ${ }^{1}$, P. Mikheenko ${ }^{2}$, T. H. Johansen ${ }^{2,3}$ and P. Kováć ${ }^{4}$

${ }^{1}$ Instituto de Ciencia de Materiales de Aragón (ICMA), CSIC - Universidad de Zaragoza, C/ María de Luna 3, 5018 Zaragoza, Spain

${ }^{2}$ Department of Physics, University of Oslo, P.O. Box 1048 Blindern, 0316 Oslo, Norway

${ }^{3}$ Institute for Superconducting and Electronic Materials, University of Wollongong, Northfields Avenue, Wollongong, NSW 2522, Australia

${ }^{4}$ Institute of Electrical Engineering, Slovak Academy of Sciences, Dúbravska cesta 9, 841 04, Bratislava, Slovakia

*Corresponding author: elenamar@unizar.es

\section{DOI: $10.1016 /$ j.jallcom.2017.05.083}

Keywords: superconductor, $\mathrm{MgB}_{2}$, critical currents, magneto-optical imaging

\begin{abstract}
:
The effects of the precursor milling energy on the superconducting and microstructural homogeneity of Fe-sheathed $\mathrm{MgB}_{2}$ monocore wires and tapes have been investigated. The conductors were produced by the powder-in-tube method using drawing and rolling deformation methods and in situ reaction. Combined magnetooptical, structural and magnetization measurements were performed on $\mathrm{MgB}_{2}$ superconducting tapes prepared from differently milled precursors revealing hidden mechanisms controlling their critical current density. Local degradation of the superconducting properties observed in some analysed conductors has been identified and correlated with microstructural observations.
\end{abstract}




\section{Introduction}

$\mathrm{MgB}_{2}$ conductors have been the subject of intense research in the last years to improve the superconducting properties and optimize the manufacturing process towards the development of homogeneous and long conductors with the performance required for power applications. With the critical temperature $T_{\mathrm{c}} \sim 39 \mathrm{~K}$, this superconductor allows operating temperatures about $20 \mathrm{~K}$, which implies enhanced thermal stability of superconducting devices and lower operating cost than for systems based on low temperature superconductors, such as $\mathrm{NbTi}$ and $\mathrm{Nb}_{3} \mathrm{Sn}$, typically working at temperatures below $5 \mathrm{~K}$. On the other hand, compared with available high $T_{\mathrm{c}}$ superconductors (HTS) based on $\mathrm{YBa}_{2} \mathrm{Cu}_{3} \mathrm{O}_{7-\mathrm{x}}$ (YBCO) and $\mathrm{Bi}_{2} \mathrm{Sr}_{2} \mathrm{Ca}_{2} \mathrm{Cu}_{3} \mathrm{O}_{12+\mathrm{x}}$ (Bi2223), the low manufacturing cost and cheap constituent materials of $\mathrm{MgB}_{2}$ conductors can open market niches in applications such as Magnetic Resonance Imaging, MRI [14], Superconducting Magnetic Energy Storage, SMES [5] or superconducting wind turbines [6].

Highly uniform and technologically reproducible critical current density, $J_{\mathrm{c}}$, in $\mathrm{MgB}_{2}$ conductors is compulsory for advanced applications. To achieve this goal, a homogeneous microstructure and phase composition distribution along the superconducting wire is required. Grain refinement of $\mathrm{MgB}_{2}$ would improve homogeneity and increase the critical current of the material due to larger density of grain boundaries, which would provide additional pinning [7,8]. Very fine grained microstructure has been widely searched by mechanical ball-milling of different precursor powders: $\mathrm{MgB}_{2}$ [9], $\mathrm{Mg}+\mathrm{B}$ [10-14], B [15-18], or Mg [12], to prepare bulks and wires with the ex situ and in situ reaction methods.

By increasing the milling energy transferred to the precursor, the irreversibility and upper critical fields are also increased, which causes a significant enhancement of $J_{\mathrm{c}}$ in the high field region $[12,13,14,16,18]$. This is a result of a poorer crystallinity of superconducting grains that induces lattice strain but also causes a small reduction of $T_{\mathrm{c}}$ $[15,18,19]$. Häßler et al [13] have correlated the observed $J_{c}(H)$ enhancement at low temperatures and high fields with the milling energy, which depends on the rotation speed and milling time, and found a transition from grain size reduction to mechanical alloying at milling energy of about $10^{8} \mathrm{~J} \mathrm{~kg}^{-1}$.

On the other hand, milling procedures may also produce some undesirable effects such as a decrease of $J_{\mathrm{c}}$ values at low magnetic fields $[14,15,16,18]$, higher oxygen content $[16,18]$ and, in some cases, a degradation of the deformation properties during wire manufacture $[13,14]$. In order to further explore the reasons of such a decrease in $J_{\mathrm{c}}$ in the low field range, we have analysed the local magnetic flux distribution inside $\mathrm{MgB}_{2}$ tapes made from different ball-milled powders using magnetooptical (MO) imaging. This technique, which is based on the rotation of the polarization plane of light passing through a magnetized ferrimagnetic garnet film, has been 
previously used to visualize the magnetic flux distribution in HTS [20-25] and $\mathrm{MgB}_{2}$ [26-27] superconductors.

In this contribution, local superconducting properties obtained by MO imaging have been correlated with magnetization measurements and microstructural observations by scanning electron microscopy (SEM), for in situ-processed $\mathrm{MgB}_{2}$ conductors made from different energy milled precursors.

\section{Experimental details}

Monocore Fe-sheathed $\mathrm{MgB}_{2}$ wires were prepared by the powder-in-tube (PIT) method using the in situ reaction technique. The starting materials were amorphous B (99\%, New Metal \& Chemicals Ltd, mean particle size lower than $1 \mu \mathrm{m}$ ) and $\mathrm{Mg}$ (Goodfellow $99.8 \%$ purity with grain size lower than $250 \mu \mathrm{m}$ ). $\mathrm{Mg}$ and B powders in stoichiometric proportions 1:2 were mixed in a vibratory mill Retsch MM 200 for 30 minutes. Afterwards, some of these precursor powders were milled in Ar atmosphere using a planetary ball mill (Retsch PM 100), with sun disk of radius $r_{\mathrm{p}}=7 \mathrm{~cm}$ and tungsten carbide (WC) jar with inner radius $r_{v}=5.25 \mathrm{~cm}$. The ball-to-powder mass ratio, $\beta$, was kept at $\sim 36$. During the milling process, one-minute pauses and change in rotation direction every three minutes were chosen to avoid local heating and formation of $\mathrm{MgB}_{2}$ by mechanical alloying. The effective ball milling time $(t)$ and rotation speed $\left(\omega_{\mathrm{p}}\right)$ are given in table 1 for each analysed wire. Three milling conditions were used: M1 (200 rpm and $t=1.5 \mathrm{~h}$ ), M2 (400 rpm and $t=3 \mathrm{~h}$ ) and M3 (400 rpm and effective milling time of $16.5 \mathrm{~h}$ ).

Fe tubes $(99.5 \%)$ of $5 \mathrm{~mm}$ outer diameter and $0.25 \mathrm{~mm}$ wall thickness were hand-filled with the different precursor powders and sealed at both ends. The diameter was reduced by drawing in consecutive steps down to the final wire diameter of 1.1 $\mathrm{mm}$. An intermediate annealing during drawing was performed to reduce the $\mathrm{Fe}$ sheath's work hardening $\left(550^{\circ} \mathrm{C}\right.$ for $1 \mathrm{~h}$ in $\left.\mathrm{Ar}\right)$. Since MO imaging requires flat surface, some pieces of these wires were rolled to tapes (of $\sim 2 \mathrm{~mm}$ width and $\sim 0.4 \mathrm{~mm}$ thickness) before the final annealing. Finally, pieces of length $\sim 10-13 \mathrm{~cm}$ were sealed to prevent oxidation, and heat treated at $670{ }^{\circ} \mathrm{C}$ for $5 \mathrm{~h}$ in vacuum $(0.1 \mathrm{mbar})$ to form the superconducting $\mathrm{MgB}_{2}$ phase. The main characteristics of the analysed wires, including a reference sample with unmilled precursor (M0), are collected in Table 1.

The microstructure and phase composition of the final wires and tapes were analysed by field-emission scanning electron microscopy (FE-SEM, Carl Zeiss MERLIN), with $15 \mathrm{kV}$ accelerating voltage and probe current of $600 \mathrm{pA}$. The phase composition was analysed using the X-ray diffraction (XRD) patterns collected at room temperature on a RIGAKU D-max /2500 X-ray diffractometer using $\mathrm{Cu} \mathrm{K}_{\alpha}$ radiation. 
Table 1. Manufacturing characteristics and properties of the analysed Fe- $\mathrm{MgB}_{2}$ wires and tapes: $t$ is the effective ball milling time, $\omega_{\mathrm{p}}$ is the mill rotation speed and $E_{\mathrm{t}} / \mathrm{m}$ is the estimated energy per unit mass transferred to the precursor (eq. 1). $T_{\mathrm{c}}$ and $\Delta T_{\mathrm{c}}$ are the critical temperature and the transition width, respectively, as derived from $\chi_{\mathrm{ac}}(T)$ measurements of the wires.

\begin{tabular}{|c|c|c|c|c|c|c|}
\hline \multirow{2}{*}{ Wire } & \multirow{2}{*}{ Tape } & \multicolumn{3}{|c|}{ Ball milling } & \multirow{2}{*}{$\begin{array}{c}T_{\mathrm{c}} \\
(\mathrm{K})\end{array}$} & \multirow{2}{*}{$\begin{array}{l}\Delta T_{\mathrm{c}} \\
(\mathrm{K})\end{array}$} \\
\hline & & $t(\mathrm{~h})$ & $\omega_{\mathrm{p}}(\mathrm{rpm})$ & $E_{\mathrm{t}} / \mathrm{m}\left(\mathrm{J} \mathrm{kg}^{-1}\right)$ & & \\
\hline W-M0 & T-M0 & 0 & 0 & 0 & 37.5 & 0.7 \\
\hline W-M1 & T-M1 & 1.5 & 200 & $1.2 \times 10^{6}$ & 37.2 & 0.6 \\
\hline W-M2 & T-M2 & 3.0 & 400 & $2 \times 10^{7}$ & 35.0 & 0.5 \\
\hline W-M3 & T-M3 & 16.5 & 400 & $1 \times 10^{8}$ & 33.0 & 1.0 \\
\hline
\end{tabular}

Vibrating sample magnetometers (VSM, Quantum Design PPMS-9T and PPMS14T) were used to measure isothermal magnetic hysteresis loops $M(H)$. AC magnetic susceptibility as a function of temperature, $\chi_{\mathrm{ac}}(T)$, measured with a SQUID-based Quantum Design MPMS-5T, was used to estimate the critical temperature $T_{\mathrm{c}}$, defined as the diamagnetism onset, and the transition width $\Delta T_{\mathrm{c}}$, defined as the change of $\chi_{\mathrm{ac}}$ inphase component $\left(\chi^{\prime}\right)$ from $10 \%$ to $90 \%$ of its value at $5 \mathrm{~K}$. For this magnetic characterisation, the iron sheath was removed by mechanical polishing because its strong ferromagnetic signal hinders the superconductor's contribution analysis. AC and DC fields were applied perpendicular to the axis of $\sim 4-5 \mathrm{~mm}$ long wires. Electrical DC transport measurements in perpendicular DC magnetic fields were performed in some wires with the 6-cm long wire immersed in liquid helium. The critical current densities, $J_{\mathrm{c}}$ were determined by the standard four-probe method using the $1 \mu \mathrm{V} / \mathrm{cm}$ criterion.

Magnetic flux distribution was visualised using MO imaging technique. As field sensor we used in-plane magnetized bismuth-substituted iron garnet sensor films, as described in $[20,21,23,25,28]$, which provide strong rotation in a weak magnetic field and give a desirable sensitivity and spatial resolution for monitoring local magnetic field on the surface of the films. The MO indicator film is placed directly on top of the polished surface of the sample and the light is reflected from the mirror on the bottom of the film before going into the analyser of the optical system set above the sample holder. The brightness of the images obtained with crossed polarizers represents the absolute value of the magnitude of the z-component of local magnetic field in the sample.

We used a polarized-light microscope and a charge coupled device (CCD) to capture and process the digital images obtained in the experiments. The microscope consists of a stabilized light source, a polariser, an analyser and optical components to project the image plane into the CCD camera with various magnifications. All optical components, especially the objective lenses, have small Verdet constants [24] to not depolarize the light beam. The magneto-optical measurements were carried out in 
reflective mode with sensitivity defined by the thickness of the magneto-optical layer the light is going through.

\section{Results and discussion}

\subsection{Effect of ball milling on the global properties of $M g B_{2}$ conductors}

The energy per unit mass transferred to the powder by ball milling, $E_{\mathrm{t}} / m$, is a useful parameter to analyse the milling effects. There are different approaches to determine $E_{\mathrm{t}} / m$. In this work we have used the formula developed in [13], which depends explicitly on the milling parameters defined in section 2 :

$$
\frac{E_{t}}{m}=c \beta \frac{\left(\omega_{p} r_{p}\right)^{3}}{r_{v}} t
$$

where $c$ is a dimensionless constant on the order of $0.1, \beta$ the ball-to-powder mass ratio, $\omega_{\mathrm{p}}$ the rotation speed, $r_{\mathrm{p}}$ the sun disk radius, $r_{v}$ the jar inner radius and $t$ the effective ball milling time. It must be noted that an unknown fraction of this energy is lost to heat, to plastic deformation of the powders or even to form $\mathrm{MgB}_{2}$ by mechanical alloying. According to Eq. (1), the used milling conditions M1, M2 and M3 described above correspond to energy values $E_{\mathrm{t}} / m \approx 1.2 \times 10^{6}, 2 \times 10^{7}$ and $1 \times 10^{8} \mathrm{~J} \mathrm{~kg}^{-1}$, respectively.

XRD pattern of the precursor powder with milling conditions M3 indicates the presence of already reacted $\mathrm{MgB}_{2}$ (mechanical alloying), as shown in Figure 1, in agreement with [13], whereas for the other milling conditions only the peaks corresponding to $\mathrm{Mg}$ are visible. Boron is in amorphous phase and therefore it is not visible in the XRD patterns. After the heat treatment of the final wire, the main phase in all wires corresponds to the superconducting $\mathrm{MgB}_{2}$. The main secondary phase is $\mathrm{MgO}$, and also WC in the wires made from milled powders.

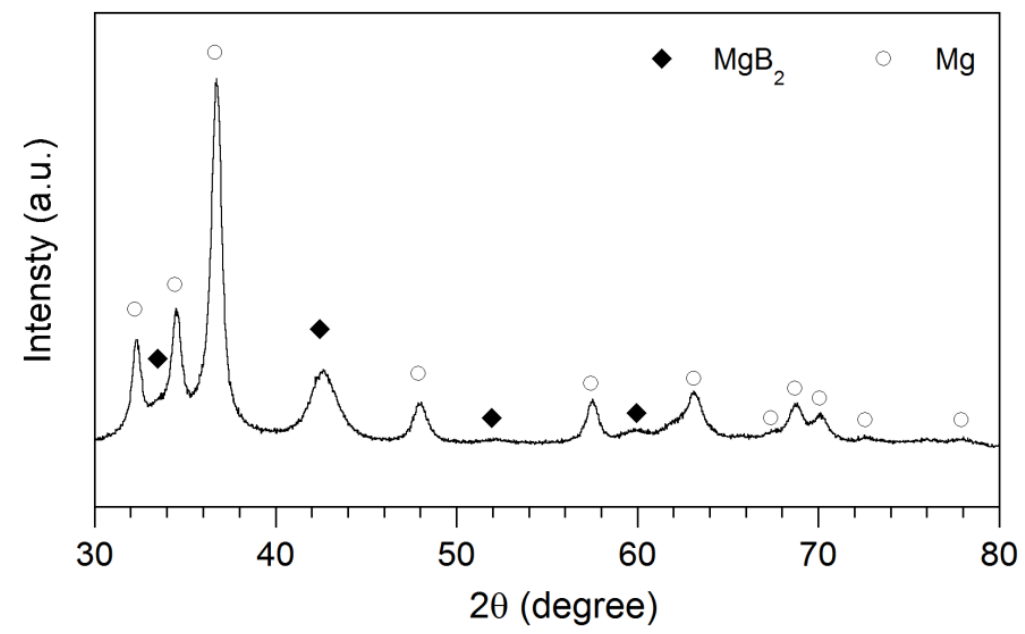

Figure 1 XRD pattern of the precursor powder with milling conditions M3. 
The precursor milling conditions affect $T_{\mathrm{c}}$ and $J_{\mathrm{c}}$ values. As shown in Table $1, T_{\mathrm{c}}$ decreases when the milling energy increases, but the transition remains always sharp, with $\Delta T_{\mathrm{c}}$ values in a range between 0.5 and $1 \mathrm{~K}$ for all wires. Figure 2 displays $J_{\mathrm{c}}(H)$ at 5 and $20 \mathrm{~K}$ of wires made with unmilled and milled powders. The values were obtained from the width of the hysteresis loop, $\Delta M(H)$, measured at constant temperature, using the equation: [29]

$$
J_{c}(H)=\frac{3 \pi}{8} \frac{\Delta M(H)}{R},
$$

which is valid for wires of radius $R$ and length $L, L \gg R$, under magnetic fields perpendicular to their axis. Magnetic flux jumps limit the magnetization values at low magnetic fields (below $2-3 \mathrm{~T}$ at $5 \mathrm{~K}$ for all wires and below $1.4 \mathrm{~T}$ at $20 \mathrm{~K}$ for $\mathrm{W}-\mathrm{M} 0$ ) leading to underestimations of $J_{\mathrm{c}}$ values in this field range, which are not displayed. The two opposite effects of ball milling on $J_{\mathrm{c}}$ are clearly seen in Figure 2. As mentioned before, on the one hand, higher energy milling enhances vortex pinning at high fields, so that the highest $J_{\mathrm{c}}$ 's at $5 \mathrm{~K}$ and $\mu_{0} H>5 \mathrm{~T}$ correspond to $\mathrm{W}-\mathrm{M} 3$. On the other hand, at low fields and higher temperatures $\left(20 \mathrm{~K}\right.$ and $\left.\mu_{0} H<3 \mathrm{~T}\right), J_{\mathrm{c}}$ decreases with increasing milling energy, which indicates poorer grain connectivity and/or larger amounts of microstructural defects than in the unmilled sample. Measurements of transport $J_{\mathrm{c}}$ of wires W-M0 and W-M1, gave values of $6 \times 10^{7} \mathrm{Am}^{-2}$ at $4.2 \mathrm{~K}$ and $8 \mathrm{~T}$ for W-M1 and 4-5 $\times 10^{7} \mathrm{Am}^{-2}$ for W-M0 (for different parts of the same wire).

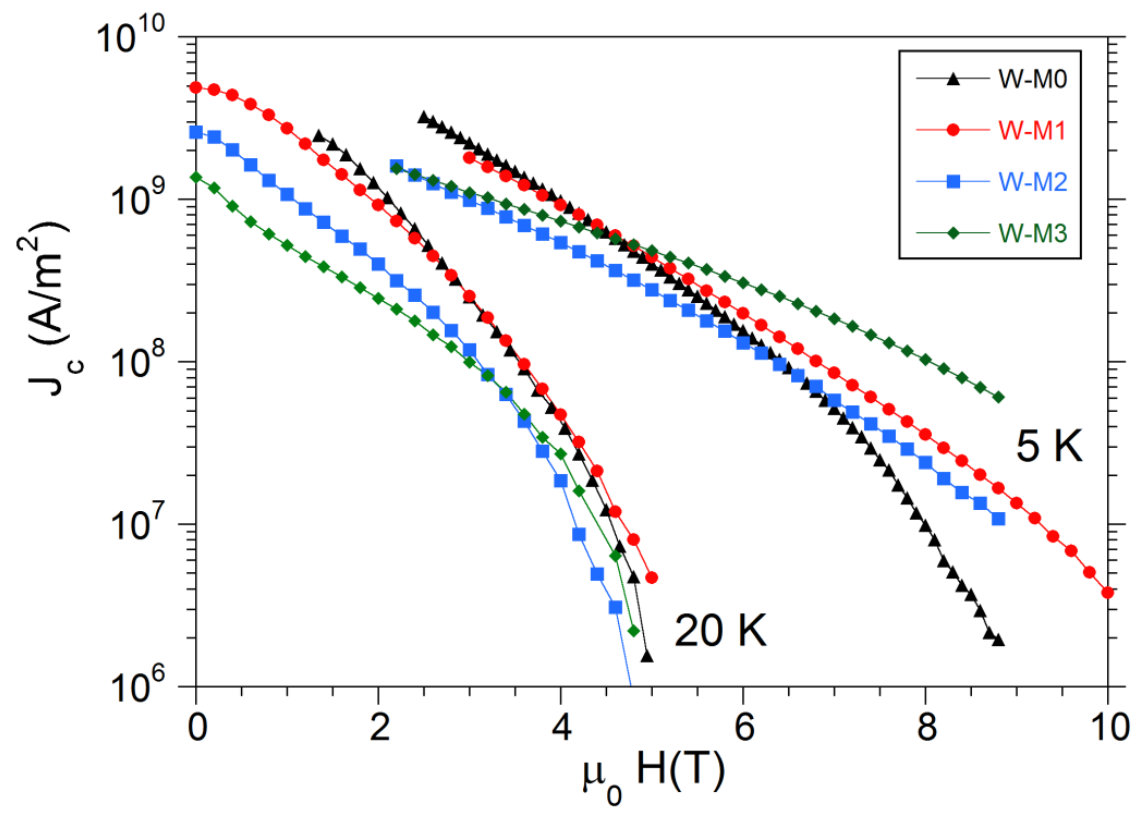

Figure 2 Magnetic field dependence of the inductive critical current density at 5 and $20 \mathrm{~K}$ for wires made from different ball milled precursors as specified in Table 1.

\subsection{Microstructural and Magneto-Optical analysis}

Magneto-optical imaging was performed to evaluate the homogeneity of the superconducting properties across and along the conductors. As mentioned previously, 
this analysis requires flat surfaces, so it has been used for characterising the tapes. One of the tapes' faces was polished, as described in [27] for Ni sheath, to remove the Fe sheath, so that there is not magnetic shielding by the ferromagnetic Fe sheath under perpendicular fields and gives direct access to the superconducting core both in MO experiments and microstructural SEM analysis.

Figure 3 shows SEM and MO images of tapes made from powders with increasing milling energies from top to bottom. SEM and MO images correspond to the same area of the sample, except in Fig. 3(d) where different areas of the same tape were analysed due to technical reasons. SEM images were taken with the secondary electrons (SE) detector to analyse the topographical differences among the samples. MO images were taken after zero-field cooling (ZFC) the sample from a temperature above $T_{\mathrm{c}}$ down to $20 \mathrm{~K}$, then applying a field of $85 \mathrm{mT}$ perpendicular to the sample surface and finally reducing the field to zero, to show the remnant flux density. It should be noted that the length of the tapes analysed by MO imaging is about five times larger than the core width ( $\sim 10$ and $\sim 2 \mathrm{~mm}$, respectively) in all samples. MO images shown in Figure 3 reveal that the maximum applied field of $85 \mathrm{mT}$ is below the field of full penetration, $H_{\mathrm{p}}$, at $20 \mathrm{~K}$ in all samples. For example, $M(H)$ curves measured in different pieces of the same tapes, gave estimations of $\mu_{0} H_{\mathrm{p}} \sim 800 \mathrm{mT}$ and $\sim 400 \mathrm{mT}$ for T-M1 and T-M2, respectively.

As reported previously [14], upon increasing the milling energy, the microstructure of the superconducting cores changes gradually, forming smaller grains and voids, but also producing variations on the voids orientation. The SEM images of the tapes shown in Figure 3 follow the same trend. In the specimen made from unmilled powders (Figure 3(a)-left), elongated voids parallel to the tape axis, with size of several hundred microns, are observed. The reason for such a microstructure is that $\mathrm{Mg}$ grains are deformed into elongated shapes during drawing and rolling due to their higher plasticity than boron. During the subsequent heat treatment, the $\mathrm{Mg}$ diffusion into nearby boron grains and the higher density of $\mathrm{MgB}_{2}$ compared to the starting powders produce elongated voids. The shape of the magnetic flux front obtained by MO imaging, Figure 3(a)-right, follows this porous structure. It must be remarked that the orientation and shape of the voids in this sample facilitates the magnetic flux penetration from both left and right ends towards the centre, compared to the other analysed samples. Note that the upper and the lower bright horizontal lines observed along the edges of tapes in MO images correspond to the MO contrast from the edges of Fe-sheath.

The SEM images shown in Figure 3 indicate that the use of precursors with increasing milling energy decreases gradually the porosity of the final tape. The MO image of the tape made with low-energy milled precursors, T-M1, Figure 3(b)-right, shows good and homogeneous superconducting properties. Note that during polishing of this tape, some Fe particles from the tape's sheath were dragged towards the superconducting core surface (light grey particles in SEM, Figure 3(b)-left). 
(a) T-M0 (unmilled)

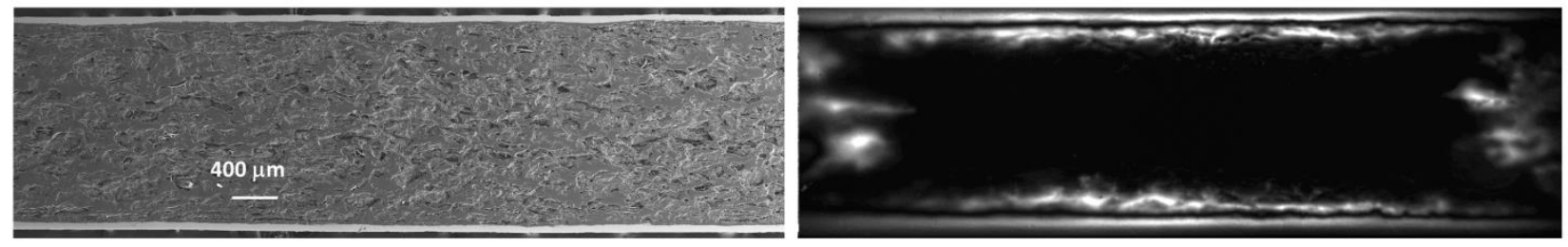

(b) T-M1 (200 rpm, $1.5 \mathrm{~h})$

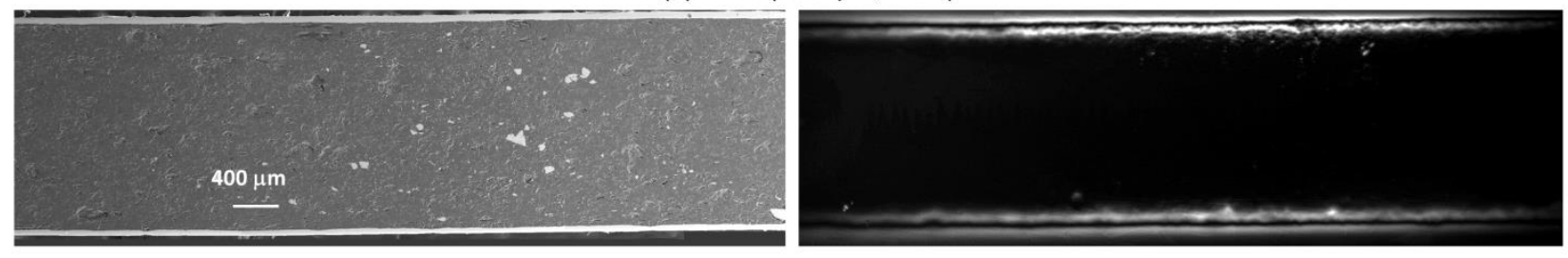

(c) T-M2 (400 rpm, $3 \mathrm{~h})$
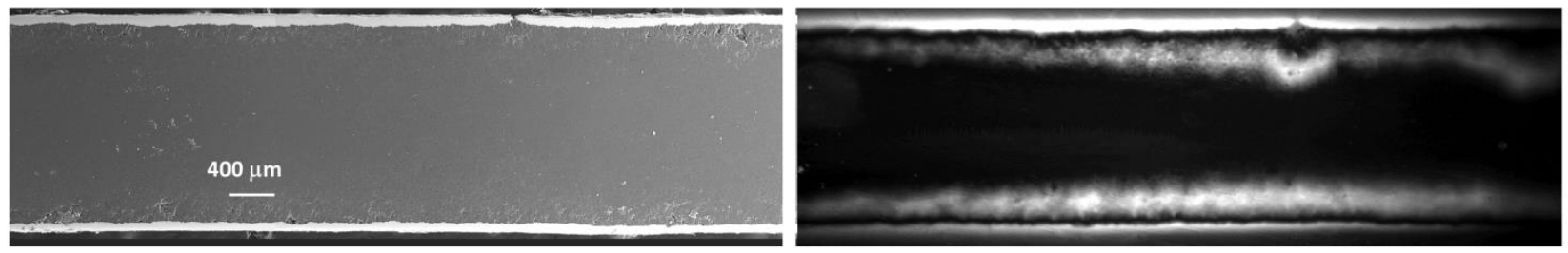

(d) T-M3 (400 rpm, $16.5 \mathrm{~h})$
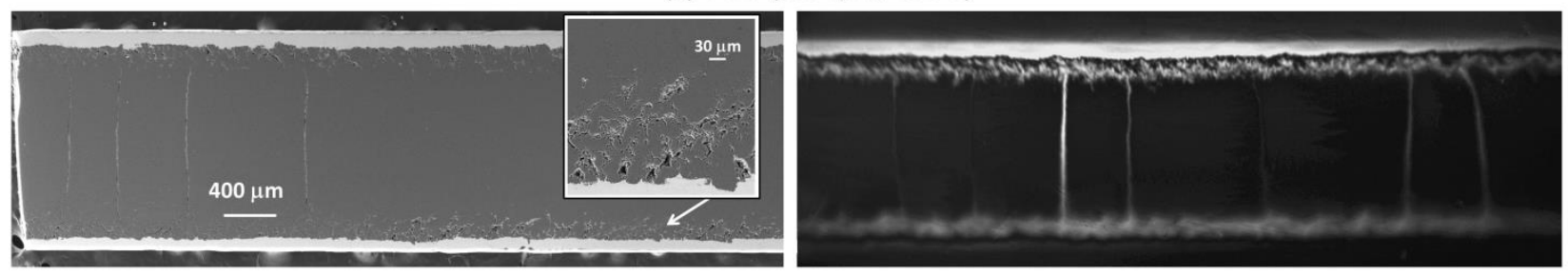

Figure 3 SEM (left) and corresponding MO images (right) of different tapes made from powder with increasing milling energies from top to bottom. SEM images were taken with the secondary electrons detector. MO images were taken after ZFC to $20 \mathrm{~K}$, then applying a field of $85 \mathrm{mT}$ perpendicular to the tape and finally removing the field. SEM and MO images correspond to the same area, except in (d) where images of different areas of the same tape were analysed. The inset in (d) shows a magnified image of the core near the sheath edge. The same magnification was used for both SEM and MO images.

Tape T-M2, Figure 3(c)-left, has a dense core but also some microstructural defects, such as pores in the Fe sheath (seen in the upper-side of the image), which affect significantly the superconducting properties of the core around, as it is seen in Fig 3(c)-right. This sample also has a region with lower local $J_{\mathrm{c}}$ (deeper penetration of the magnetic flux) in the upper-right part of the image, which, again, coincides with a microstructural defect.

Finally, the tape made from the highest energy milled precursor (T-M3) has cracks perpendicular to the long direction of the tape, as clearly seen in Figure 3(d)-left. MO image (Figure 3(d)-right) shows also deep magnetic flux penetration along these cracks that cross the superconducting core and strongly decrease $J_{\mathrm{c}}$ in this tape. During mechanical deformation, the fine-milled precursor forms highly dense blocks separated 
by less-denser packed powders or even cracks that cross the tape from side to side perpendicular to it. This forms the brick-like structures that cannot be restored by the final heat treatment. Moreover, SEM images show that the core is very dense in the centre of this tape, while at the edges (upper and lower areas in Figure 3(d)-left), microstructural defects such as cracks and voids are present, which produce magnetic flux patterns with ripple-like shape, as shown in Figure 3(d)-right.

Since the reasons of the observed deterioration of superconducting properties in the sample T-M2 were unknown, further MO experiments and SEM analysis were performed, comparing it with tape T-M1, which shows the best behaviour among the analysed tapes. With this purpose, MO images were recorded with the following thermal and magnetic history: the tape was field-cooled (FC) from $T>T_{\mathrm{c}}$ in a perpendicular field of $17 \mathrm{mT}$ down to $20 \mathrm{~K}$. Next, after stabilizing the temperature, the field was decreased to zero. Subsequently, the field amplitude was increased again but in the opposite direction to $-85 \mathrm{mT}$, and finally returned back to zero. The results for tapes T-M2 and T-M1 are shown in Figures 4 and 5, respectively. The $M(H)$ curves for similar magnetic history, but measured in different pieces of the same tapes, are also shown. MO images taken in a field-cooled sample following this specific magnetic field protocol highlights the distortions in the magnetic pattern so that it is helpful to reveal inhomogeneities in the superconductor.

Figure 4 shows MO images of tape T-M2 taken at different magnetic fields during this measurement. The image (not shown) taken just after field cooling the sample to $20 \mathrm{~K}$ in $17 \mathrm{mT}$, displays a uniform grey background. Upon decreasing the field to zero (b) a magnetic flux inhomogeneity (area marked by an arrow) becomes visible in the MO image, and gets clearer when further increasing the field amplitude in the opposite direction, as shown in images (c) and (d). Note that at the magnetic field of $-85 \mathrm{mT}$, shown in (d), the shape of the penetration front is similar to that in Figure 3(c). When slightly decreasing the amplitude of the field again to $-76.5 \mathrm{mT}$, image (e), some new features in the penetration field front appear suddenly in the upper part of the image (at positions marked with continuous arrows), and also some "bubble like shape" magnetic inhomogeneities are formed in the central part of the sample (marked by dotted arrows). It must be noted that the initial round inhomogeneity is always observed in the same position in all measurement runs performed both at 5 and $20 \mathrm{~K}$. While the bubble-like feature, always suddenly formed in the same area, is not always of the same structure. This behaviour suggests the existence of poor superconducting connectivity between regions in this part of the sample. Finally, MO image (f) shows the trapped field, which has similar pattern as in (e) but with the expected dark-bright-dark profile revealing the existence of flux-antiflux areas due to hysteresis and the performed thermal-magnetic history. It must be remarked that the left part of this sample shows good homogeneity and better superconducting properties than the centre and right part of the sample. 

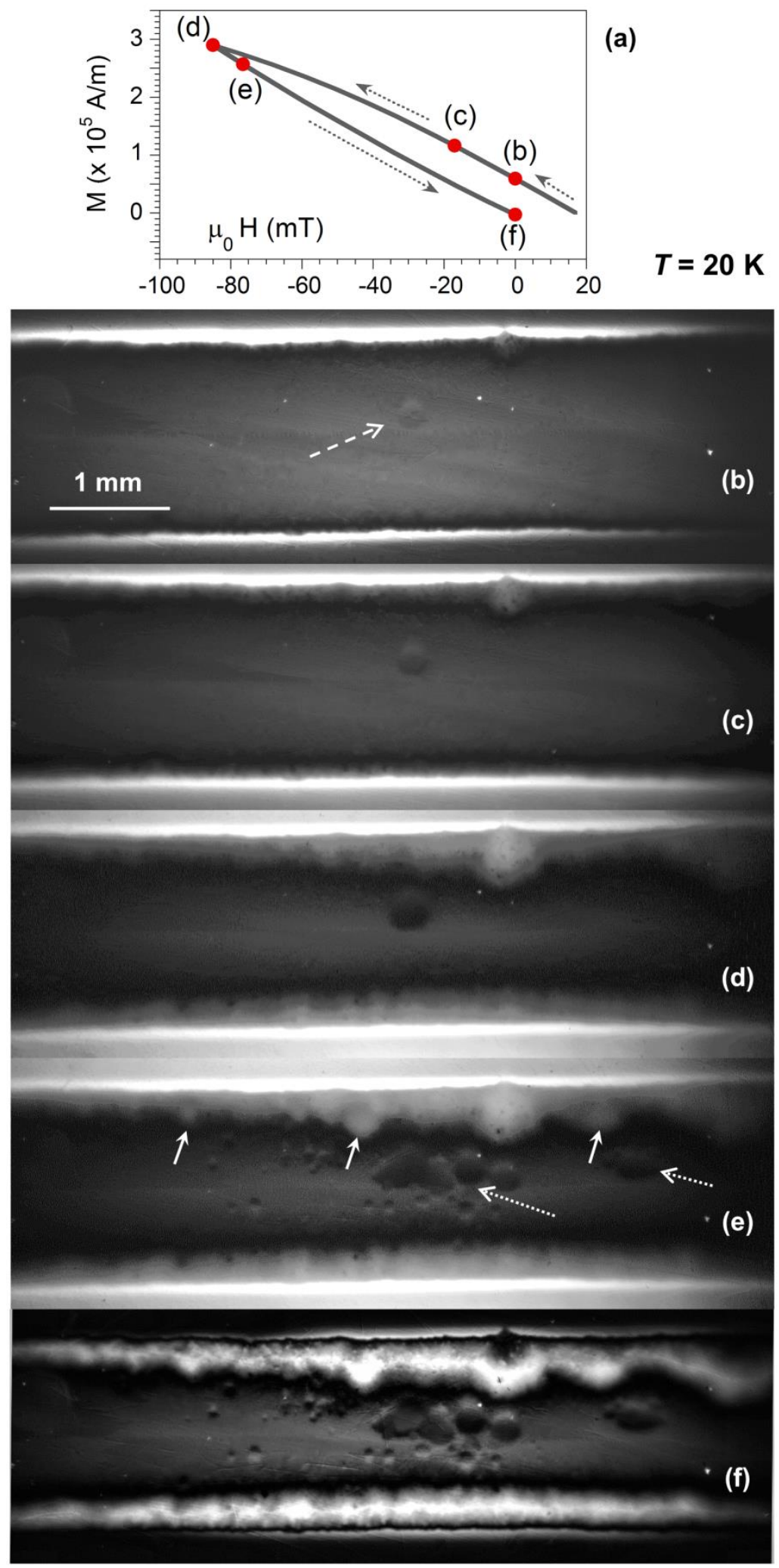

Figure 4 (a) Magnetization values, $M(H)$, and (b)-(f) MO images of the tape T-M2 at different fields after FC in $17 \mathrm{mT}$ down to $20 \mathrm{~K}$, then changing the field to $-85 \mathrm{mT}$ and finally removing the field. The images correspond to: (b) $0 \mathrm{mT}$, (c) $-17 \mathrm{mT}$, (d) $-85 \mathrm{mT}$, (e) $-76.5 \mathrm{mT}$ and (f) $0 \mathrm{mT}$, as marked in (a). The magnetic field is applied perpendicular to the tape. The sample of MO images is the same as in Figure 3(c), while $M(H)$ was measured in a different piece. 

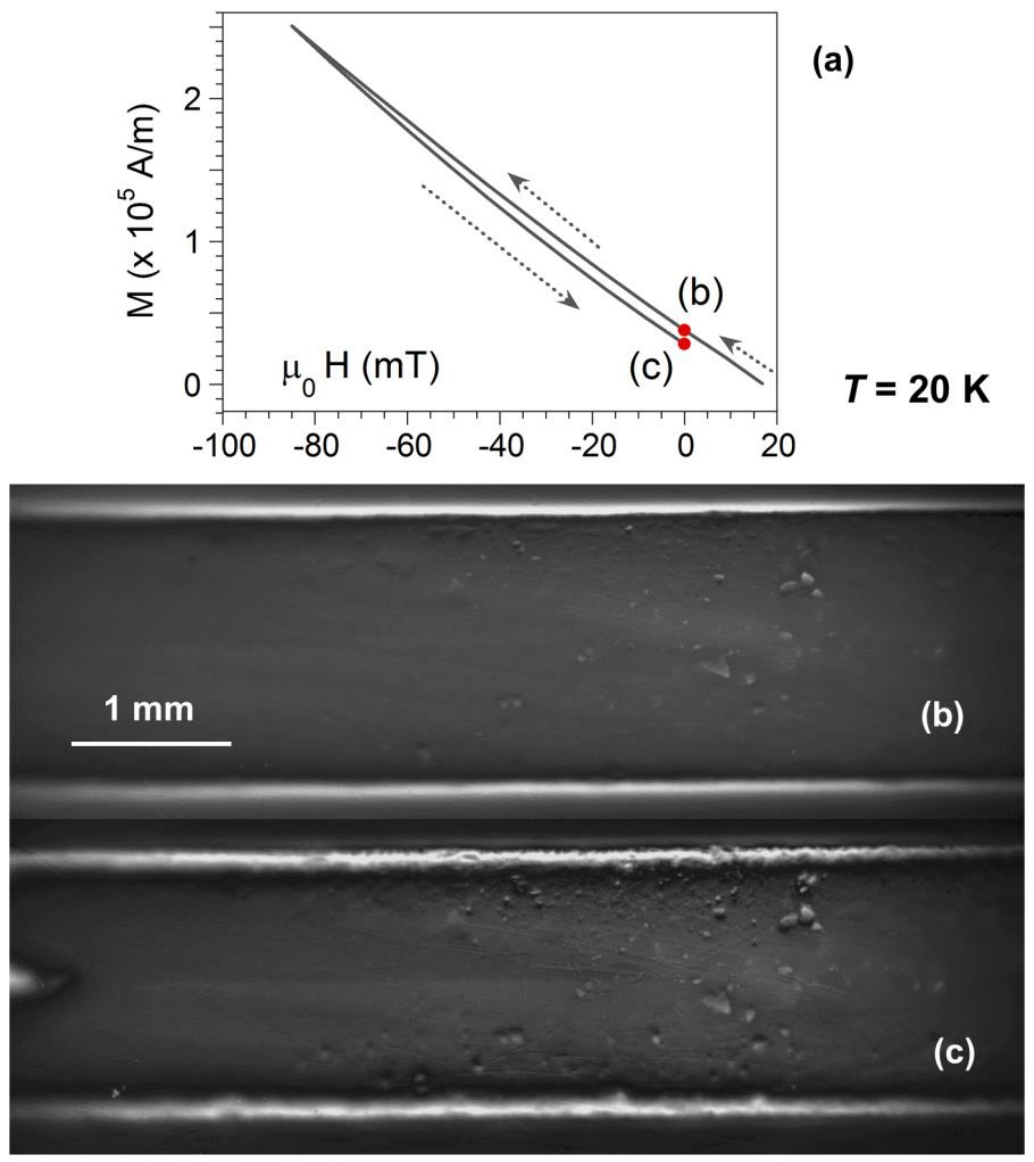

Figure 5 (a) Magnetization values, $M(H)$, and (b)-(c) MO images of the tape T-M1 in zero-field of the upper and lower branch, respectively, as marked in (a). Similar thermal and magnetic history as in Figure 4 was performed: the sample was FC in $17 \mathrm{mT}$ down to $20 \mathrm{~K}$, the field was then changed to $-85 \mathrm{mT}$ and finally returned back to zero. The sample of MO images is the same as in Figure 3(b), while $M(H)$ was measured in a different piece.

For comparison, Figure 5 shows MO images of the tape T-M1 during a similar measurement run as in Figure 4. It must be emphasised that the upper and lower branches of $M(H)$ here are very similar, indicating small penetration of the magnetic field and therefore higher $J_{\mathrm{c}}$ of T-M1 compared to T-M2. That is also in agreement with observations in ZFC measurements shown in Figure 3. This tape presents indeed a very homogeneous magnetic flux profile and therefore only both images taken in zero-field are shown: in the decreasing (b) and increasing (c) field branch. Note that the Fe particles present in the surface of the core (Figure 3(b)-left) are well visible in MO images after field-cooling the sample, but do not have any influence in the superconducting behaviour of this tape, as expected.

The magnetic inhomogeneities observed in FC MO images of tape T-M2 in Figure 4 could be due to the presence of cavities or holes, or to the existence of poor superconducting links between regions within this sample. A further analysis by SEM of this tape discards the existence of cavities, and confirms the formation of aggregates of $\mathrm{MgB}_{2}$ poorly connected with each other, which are observed in this part of the sample, as shown in Figure 6(b). The observed grain structure resembles the 
microstructure of some ex situ $\mathrm{MgB}_{2}$ materials [30]. By contrast, other areas of this tape, with higher $J_{\mathrm{c}}$ and good homogeneity according to MO images (as in the left area in Figure 4), are well connected as it is seen in Figure 6(a). It must be remarked that the SEM images of tapes made with unmilled, Figure 6(c), and milled precursors with milling conditions M1, Figure 6(d), show well-connected superconducting grains.
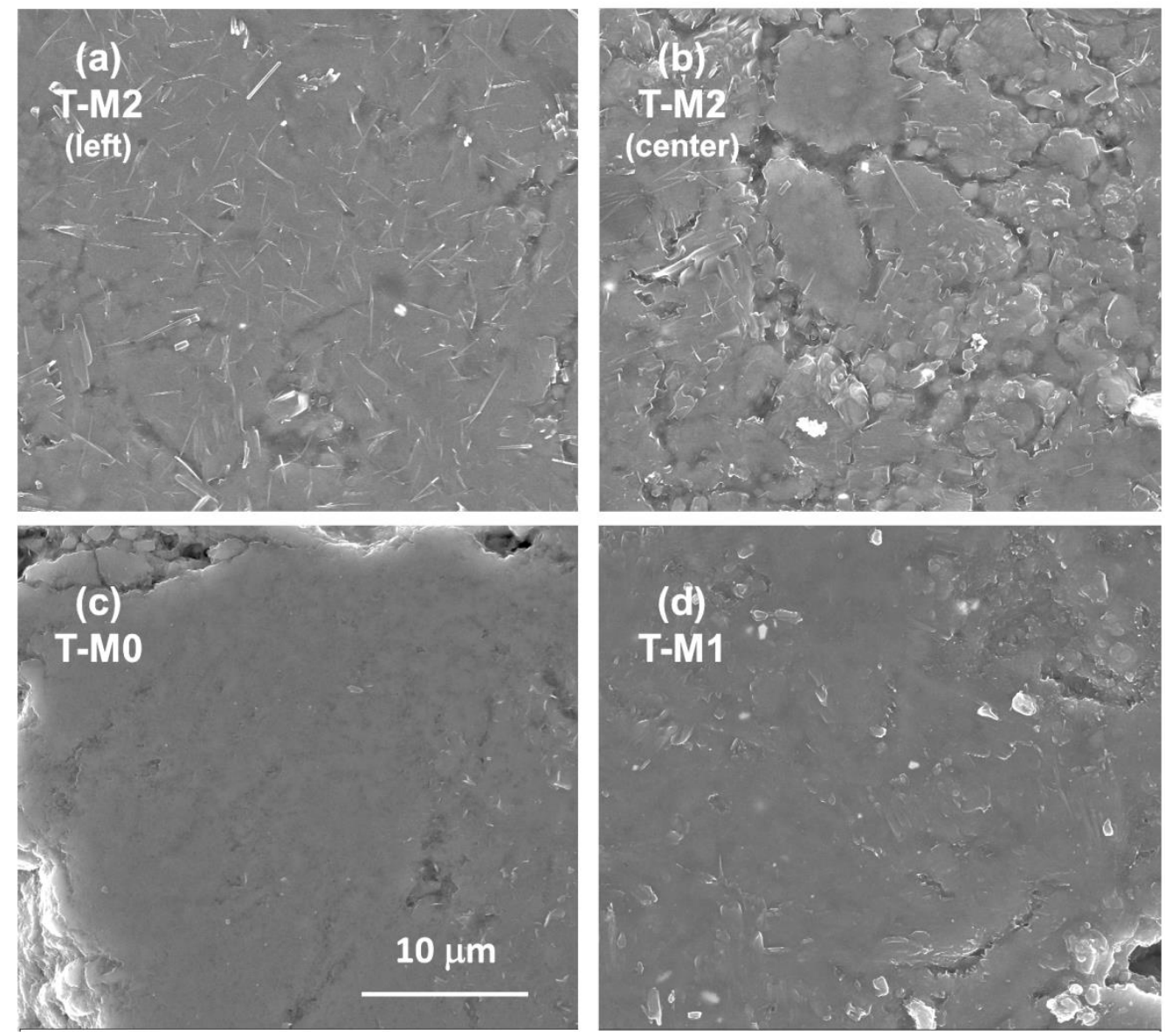

Figure 6. SEM images (secondary electrons detector) of the tape T-M 2 in the left region (a), where $J_{\mathrm{c}}$ is higher, and in the central area (b) where "bubble like shape" magnetic inhomogeneities are seen in Figure 4. Image (c) is of the tape with unmilled precursor and (d) of T-M1. The same magnification has been used in all images.

\section{Conclusions}

We have analysed the effect of precursor ball milling on the properties of monocore Fe-sheathed $\mathrm{MgB}_{2}$ wires and tapes prepared by the in situ powder-in-tube method by using drawing and rolling techniques. The effect of milling energies per unit mass in the range between $1.2 \times 10^{6}$ and $1 \times 10^{8} \mathrm{~J} \mathrm{~kg}^{-1}$ (above the mechanical alloying limit) on the superconducting properties of these conductors were analysed.

Magneto-optical (MO) imaging analysis has been performed in order to investigate magnetic flux penetration in these samples, revealing local $J_{\mathrm{c}}$ variations in these superconductors and allowing further understanding of the reasons of observed $J_{\mathrm{c}}$ 
deterioration at low magnetic fields frequently observed with increase of the milling energy of the precursor powders. It was found that microstructural characteristics change strongly with milling energy of the precursor and affect significantly the magnetic flux front propagation observed by MO imaging at zero-field-cooled and field-cooled conditions. MO imaging performed in field-cooled samples, following a specific magnetic field sequence, highlights the distortions in the magnetic pattern so that it has been found very helpful to reveal inhomogeneities in the superconductor. The MO images have been correlated with microstructural features observed with scanning electron microscopy (SEM), such as small cracks, sheath pores or weakly-linked aggregates of $\mathrm{MgB}_{2}$ grains present in some of the analysed tapes.

Ball-milling the precursor enhances vortex pinning at high magnetic fields, so that the highest $J_{\mathrm{c}}$ 's at $5 \mathrm{~K}$ and $\mu_{0} H>5 \mathrm{~T}$ correspond to the conductor made from the highest energy milled precursor. Nevertheless, the use of the precursor with milling energies above a certain limit produces microstructural defects during mechanical deformation, resulting in the formation of large transversal cracks, which indicates that using just drawing to deform the wire is not appropriate to fabricate conductors from high energy milled precursor powders. The tape fabricated from the precursor with the lowest milling energy shows good homogeneity.

\section{Acknowledgments}

This work was supported by the Spanish Ministerio de Economía y Competitividad and the European FEDER Program (Projects MAT2011-22719 and ENE-2014-52105-R), and by the Gobierno de Aragón (research group T12). We also acknowledge technical assistance of I. Cabistany and J. A. Gómez during the manufacture of the wires and tapes, and the use of Servicio General de Apoyo a la Investigación- SAI, Universidad de Zaragoza. 


\section{References}

[1] M. Razeti, S. Angius, L. Bertora, D. Damiani, R. Marabotto, M. Modica, D. Nardelli, M. Perrella and M. Tassisto, Construction and operation of cryogen free $\mathrm{MgB}_{2}$ magnets for open MRI systems IEEE Trans. Appl. Supercond. 18 (2008) 882

[2] Y. Lvovsky, E.W. Stautner and T. Zhang, Novel technologies and configurations of superconducting magnets for MRI, Supercond. Sci. Technol. 26 (2013) 093001

[3] J. Ling, V. Voccio, S. Hahn, Y. Kim, J. Song, J. Bascuñán, and Y. Iwasa, Construction and persistent-mode operation of $\mathrm{MgB}_{2}$ coils in the range $10-15 \mathrm{~K}$ for a $0.5 \mathrm{~T} / 240 \mathrm{~mm}$ cold bore MRI magnet, IEEE Trans. Appl. Supercond. 25 (2015) 4601705

[4] H.S. Kim, C. Kovacs, M. Rindfleisch, J. Yue, D. Doll, M. Tomsic, M.D. Sumption and E.W. Collings, Demonstration of a conduction cooled react and wind $\mathrm{MgB}_{2}$ coil segment for MRI applications, IEEE Trans. Appl. Supercond. 26 (2016) 4400305

[5] F. Hornung, M. Klaeser, P. Leys, and T. Schneider, Construction and test of $\mathrm{MgB}_{2}$ mockup coils for LIQHYSMES, IEEE Trans. Appl. Supercond. 26 (2016) 5700405

[6] I. Marino, A. Pujana, G. Sarmiento, S. Sanz, J.M. Merino, M. Tropeano, J. Sun and T. Canosa, Lightweight $\mathrm{MgB}_{2}$ superconducting $10 \mathrm{MW}$ wind generator, Supercond. Sci. Technol. 29 (2016) 024005

[7] S.-F. Wang, Z. Liu, Y.-L. Zhou, Y.-B. Zhu, Z.-H. Chen, H.-B. Lu, B.-L. Cheng and G.-Z. Yang, Correlation between film thickness and critical current density of $\mathrm{MgB}_{2}$ films, Supercond. Sci. Technol. 17 (2004) 1126

[8] P. Mikheenko, E. Martínez, A. Bevan and J.S. Abell and J.L. MacManus-Driscoll, Grain boundaries and pinning in bulk $\mathrm{MgB}_{2}$, Supercond. Sci. Technol. 20 (2007) S264

[9] A. Malagoli, V. Braccini, M. Tropeano, et al., Effect of grain refinement on enhancing critical current density and upper critical field in un-doped $\mathrm{MgB}_{2}$ ex situ tapes J. Appl. Phys. 104 (2008) 103908

[10] A. Gümbel, J. Eckert, G. Fuchs, K. Nenkov, K.-H. Müller, L. Schultz, Improved superconducting properties in nanocrystalline bulk $\mathrm{MgB}_{2}$, Appl. Phys. Lett. 80 (2002) 2725 .

[11] H. Fang, S. Padmanabhan, Y.X. Zhou, K. Salama, High critical current density in iron-clad MgB2 tapes, Appl. Phys. Lett. 82 (2003) 4113

[12] A. Matsumoto, H. Kumakura, H. Kitaguchi, H. Hatakeyama, H. Yamada, M. Hirakawa, The microstructures and superconducting properties of $\mathrm{MgB}_{2}$ tapes processed in-situ by a ball-milling method, IEEE Trans. Appl. Supercond. 15 (2005) 3333

[13] W. Häßler, H. Hermann, C. Rodig, A. Aubele, L. Schmolinga, B. Sailer and B. Holzapfel, Influence of the milling energy transferred to the precursor powder on the microstructure and the superconducting properties of $\mathrm{MgB}_{2}$ wires, Supercond. Sci. Technol. 26 (2013) 025005

[14] C. Laliena, E. Martínez, L.A. Angurel and R. Navarro, Effect of ball milling and fatty acid addition on the properties of $\mathrm{MgB}_{2}$ wires IEEE Trans. Appl. Supercond. 25 (2015) 6945820 
[15] X. Xu, J. H. Kim, M. S. A. Hossain, J. S. Park, Y. Zhao, S. X. Dou, W. K. Yeoh, M. Rindfleisch, and $\mathrm{M}$. Tomsic, Phase transformation and superconducting properties of $\mathrm{MgB}_{2}$ using ball-milled low purity boron, J. Appl. Phys. 103 (2008) 023912.

[16] X. Xu, J. H. Kim, S. X. Dou, S. Choi, J. H. Lee, H. W. Park, M. Rindfleish \& M. Tomsic, A correlation between transport current density and grain connectivity in $\mathrm{MgB}_{2} / \mathrm{Fe}$ wire made from ball-milled boron. J. Appl. Phys., 105 (2009) 1.

[17] B.H. Jun, N.K. Kim, K.S. Tan and C.J. Kim, Enhanced critical current properties of in situ processed $\mathrm{MgB}_{2}$ wires using milled boron powder and low temperature solid-state reaction, J. Alloys Compd. 492 (2010) 446.

[18] B.H. Jun, S.D. Park and C.J. Kim, Refinement and carbon incorporations effects on the superconducting properties of $\mathrm{MgB}_{2}$ through wet milling process of low purity boron powder, J. Alloys Compd. 535 (2012) 27.

[19] A. Yamamoto, J. Shimoyama, S. Ueda, Y. Katsura, I. Iwayama, S. Horii and K. Kishio, Universal relationship between crystallinity and irreversibility field of $\mathrm{MgB}_{2}$, Appl. Phys. Lett. 86 (2005) 212502.

[20] A.A. Polyanskii, M.V. Indenbom, Yu. A. Ossip'yan, V.K. Vlasko-Vlasov, V.I. Nikitenko, Visualization of magnetization processes in HTSCs. IEEE Trans. Magn. 26 (1990) 14451448.

[21] L.A. Dorosinskii, M.V. Indenbom, V.I. Nikitenko, Yu.A. Ossip'yan, A.A. Polyanskii, V.K. Vlasko-Vlasov, Studies of HTSC crystal magnetization features using indicator magnetooptic films with in-plane anisotropy. Physica C 203 (1992) 149.

[22] M.R. Koblischka, T.H. Johansen and H. Bratsberg, Bending of silver-sheathed (Bi,Pb)2223 tapes investigated by magneto-optical flux visualization, Supercond. Sci. Technol. 10 (1997) 693.

[23] A.A. Polyanskii, X.Y. Cai, D.M. Feldmann, D.C. Larbalestier, Visualization of magnetic flux in magnetic materials and high temperature superconductors using the Faraday Effect in ferrimagnetic garnet films", in Nano-crystalline and Thin Film Magnetic Oxides (NATO Science Series 3. High Technology vol 72) ed I. Nedkov and M. Ausloos, Kluwer Academic Publishers (1999) 353-370. (Proceedings of the NATO Advanced Research Workshop on Ferrimagnetic Nano-Crystalline and Thin Film Magnetooptical and Microwave Materials. Sozopol, Bulgaria. Sept. 27-Oct.3, 1998).

[24] Ch. Jooss, J. Albrecht, H. Kuhn, S. Leonhardt and H. Kronmüller, Magneto-optical studies of current distributions in high-Tc superconductors Rep. Prog. Phys. 65 (2002) 651-788.

[25] A.A. Polyanskii, D.M. Feldmann, D.C. Larbalestier, Magneto-Optical characterization techniques, Chapter D3.4 of Handbook of Superconducting Materials, Ed D.A. Cardwell and D.S. Ginley (IOP publishing Ltd) (2003), p. 1551-1567.

[26] D.C. Larbalestier, L.D. Cooley, M.O. Rikel, A.A. Polyanskii, J. Jiang, S. Patnaik, X.Y. Cai, D. M. Feldmann, A. Gurevich, A.A. Squitieri, M.T. Naus, C.B. Eom, E.E. Hellstrom, R.J. Cava, K.A. Regan, N. Rogado, M.A. Hayward, T. He, J.S. Slusky, P. Khalifah, K. Inumaru and M. Haas, Strongly linked current flow in polycrystalline forms of the superconductor $\mathrm{MgB}_{2}$, Nature 410 (2001)186.

[27] A Polyanskii, V Beilin, I Felner, M I Tsindlekht, E Yashchin, E Dul'kin, E Galstyan, M Roth, B Senkowicz, E Hellstrom, Magneto-optical and electromagnetic studies of core connectivity and weak-link behavior in $\mathrm{Cu} / \mathrm{MgB}_{2}$ and $\mathrm{Ni} / \mathrm{MgB}_{2}$ wires and tapes, Supercond. Sci. Technol. 17 (2004) 363. 
[28] L.E. Helseth, W. Hansen, E.I. Il'yashenko, M. Baziljevich M, and T.H. Johansen, Faraday rotation spectra of bismuth-substituted ferrite garnet films with in-plane magnetization Phys. Rev. B 64 (2001) 174406.

[29] M.N. Wilson, Superconducting magnets, Clarendon Press, Oxford, UK, (1983) 165-170.

[30] Y. Shimada, S. Hata, K. Ikeda H. Nakashima, S. Matsumura, H. Tanaka, A. Yamamoto J. Shimoyama and K. Kishio, Microstructural connectivity in sintered ex-situ $\mathrm{MgB}_{2}$ bulk superconductors, J. Alloys Compd. 656 (2016) 172. 